\title{
Evaluation of the clinical usefulness of high-frequency ultrasonography in pre-operative evaluation of cutaneous melanoma - a prospective study
}

\author{
Paweł Piłat ${ }^{1,2}$, Adam Borzęcki², Mieczysław Jazienicki ${ }^{3}$, Agnieszka Gerkowicz ${ }^{1}$, Franciszek Szubstarski ${ }^{4}$, \\ Dorota Krasowska ${ }^{1}$
}

\author{
${ }^{1}$ Chair and Department of Dermatology, Venereology and Pediatric Dermatology, Medical University of Lublin, Lublin, Poland \\ ${ }^{2}$ Non-Public Health Care Center Med-Laser, Lublin, Poland \\ ${ }^{3}$ Department of Surgical Oncology, $1^{\text {st }}$ Independent Public Clinical Hospital, Lublin, Poland \\ ${ }^{4}$ Department of Cancer Pathology, St. John of Dukla Lublin Region Cancer Center, Lublin, Poland
}

Adv Dermatol Allergol 2020; XXXVII (2): 207-213

DOI: https://doi.org/10.5114/ada.2018.79939

\begin{abstract}
Introduction: There are many papers in the available literature on the pre-operative evaluation of malignant melanoma (MM) using a $20 \mathrm{MHz}$ probe. In many Western European countries, this examination is standard in the diagnosis of such lesions in terms of the resection margin. However, few papers describe and compare the results of melanoma thickness measurements using 20 and $50 \mathrm{MHz}$ frequency probes.

Aim: The aim of the study was to evaluate the thickness of melanoma using 20 and $50 \mathrm{MHz}$ probes. In addition, the obtained values were evaluated in comparison to the thickness values obtained in the histopathological examination.

Material and methods: Melanoma malignum was confirmed histopathologically in 19 out of 72 patients. Then only those 19 patients were enrolled in the study.

Results: A correlation was demonstrated between the thickness of the melanoma measured with the 20 and $50 \mathrm{MHz}$ probes, expressed in $\mathrm{mm}$, and the thickness obtained in the histopathological examination expressed in millimeters (Breslow's scale). It was $0.759(p<0.001)$ and $0.734(p<0.001)$, respectively. The average thickness of melanomas obtained in ultrasound examination was identical for both probes and was $0.74 \mathrm{~mm}$.

Conclusions: It seems that both types of probes can be used to assess melanoma thickness. The demonstrated correlation of high-frequency ultrasonography (HFUS) thickness with Breslow's scale indicates that the HFUS evaluation can be used in determination of melanoma resection margins as a repeatable, painless, non-invasive test.
\end{abstract}

Key words: melanoma, ultrasonography, thickness.

\section{Introduction}

Early diagnosis and implementation of appropriate therapy is of great importance in improving the prognosis of patients with malignant melanoma (MM). The procedure of choice is surgical removal and histopathological examination with regard to specific parameters [1]. One of them is Breslow's scale developed by Alexander Breslow in 1970. Thickness of the melanoma along with the number of mitoses and presence or absence of ulceration shows the greatest correlation with the degree of 5-year survival [2]. So far, individual studies have addressed the issue of pre-operative melanoma evaluation using ultrasonography, high-frequency ultrasonography (HFUS) and optical coherence tomography (OCT) [3-12].
In the sonographic image, melanomas look like homogeneous, flat or elliptic structures in the subcuticular region, with clearly marked borders. However, inflammatory infiltration in the lower part of the lesion may falsely overestimate the thickness of MM. Unfortunately, currently the HFUS examination does not allow pigmented lesions to be distinguished from cutaneous melanoma. Dermoscopy is still the gold standard in the detection of early forms of cutaneous melanoma. The MM sonographic examination seems to be helpful in pre-operative evaluation of melanoma due to the appropriate selection of resection margins [3]. This is also important with respect to lymphatic vessels and lymph flow. Too wide resection margins may prevent the sentinel node from being properly identified during further diagnostics, es-

Address for correspondence: Paweł Piłat MD, PhD, Chair and Department of Dermatology, Venereology and Pediatric Dermatology, Medical University of Lublin, 13 Radziwiłłowska St, 20-080 Lublin, Poland, phone: +48 607760 179, e-mail: pawelpilat1@gmail.com Received: 27.08.2018, accepted: 8.10.2018. 
pecially in the case of MM over $1.0 \mathrm{~mm}$ thick. Disruption of lymph flow from the tumor area, created during tumor excision, prevents proper detection of the sentinel node during lymphoscintigraphy. In turn, a too narrow margin may result in an unlawful resection of the lesion. The $20 \mathrm{MHz}$ probe is the most commonly used in the pre-operative evaluation of melanoma. Probes with a frequency of 30, 75 and $100 \mathrm{MHz}$ were used in single studies [3].

\section{Aim}

The aim of the study was to assess the usefulness of high-frequency sonographic examination using 20 and $50 \mathrm{MHz}$ probes in pre-operative melanoma evaluation. A further aim was to compare the thickness obtained in ultrasound measurements and that obtained in histopathological examination.

\section{Material and methods \\ Patients}

Seventy-two patients with suspected malignant lesions were qualified for the study from April 2015 until December 2016. Two patients did not agree to continue the study. The other patients received information and gave their informed consent to participate in the study. The study was approved by the local bioethics commission (no. IWL/237/7123/2016).

\section{Research methodology}

In all patients, the study was carried out according to one schedule:

- Clinical and dermoscopic evaluation of skin lesion.

- Ultrasound examination.

- Surgical removal.

- Histopathological examination.

\section{Clinical and dermatoscopic evaluation}

Initially, each pigmented lesion was evaluated macroscopically, and photographic documentation was also made. Then a dermatoscopic examination was carried out using a DERMLITE DL3 hand dermatoscope, providing 10× magnification and photographic documentation.

\section{Ultrasound examination}

Pigmented lesions and normal skin (approximately $3 \mathrm{~cm}$ from the lesion) were evaluated with a DermaScan C ultrasound machine produced by the Danish company Cortex Technology ApS using two ultrasound probes with a frequency of $20 \mathrm{MHz}$ (resolution 60 to $200 \mu \mathrm{m}$ and $23 \mathrm{~mm}$ of penetration) and $50 \mathrm{MHz}$ (resolution $60 \mu \mathrm{m}$ and $3 \mathrm{~mm}$ of penetration). Equipment named DermaScan C is on the FDA list as a medical device in the regulatory class II/21 CFR 892.1560 (510 (k) No. (K983945). It provides real-time tissue scanning with a frequency of 6-8 frames per second.

Ultrasonography was performed after previous application of a standard sonography gel on the lesion. Demineralized water along with the appropriate film provided by the manufacturer were located between the probe and the examined lesion. During the examination, the probe was directed perpendicularly to the surface of the skin and lesions. This aimed to determine the shape of the lesion and its thickness between the lower limit of the entrance echo and the deepest lower margin (Figures 1 and 2). Ultrasonography of normal skin, approximately $3 \mathrm{~cm}$ from the lesion, was also performed in each patient.

\section{Histopathological examination}

All cancer lesions were surgically removed with an appropriate margin, appropriate for the type of lesion. According to the current guidelines applicable in Poland for patients suspected to have malignant melanoma, excision of lesions should take place with a margin of 1-3 mm. Than after the histopathological examination and the final thickness is obtained, re-excision with the appropriate margin is performed (Table 1) [13]. The removed lesions were subjected to histopathological examination and were evaluated taking into account, inter alia, Breslow's and Clark's scales and the number of mitoses. The pathomorphologist was not informed about the thickness of the removed lesion obtained in the sonographic examination.

\section{Results}

In the examined group of 72 patients, 19 patients were finally diagnosed with cutaneous melanoma. In the further part of the study, 19 patients with cutaneous melanoma, including 13 women and 6 men, were included in the study group. The mean age of the patients was 57 years (median: 58 years). The youngest respondent was 26 years old, while the oldest was 85 years old. The standard deviation indicates that age differences were greater among women than men (Table 2). Of the 19 malignant lesions, 4 nodular melanomas (NM) and 8 superficial spreading melanomas (SSM) were identified, of which 2 were nevi, 6 malignant lentigines and 1 melanoma in regression.

The average thickness of the tumor on Breslow's scale was $0.56 \pm 0.47 \mathrm{~mm}$ (Table 3 ). On Clark's scale, 9 patients had grade I infiltration, grade II was found in 7 patients, and there was one case each with grade III, IV and V infiltration (Table 4). The average thickness of cancer lesions for 20 and $50 \mathrm{MHz}$ ultrasound probes was the same and was $0.74 \mathrm{~mm}$. The number of lesions assessed as thicker than $1 \mathrm{~mm}$ with the $20 \mathrm{MHz}$ probe was the same as in the case of the $50 \mathrm{MHz}$ probe $(n=4)$. Also the number of lesions evaluated in the study 

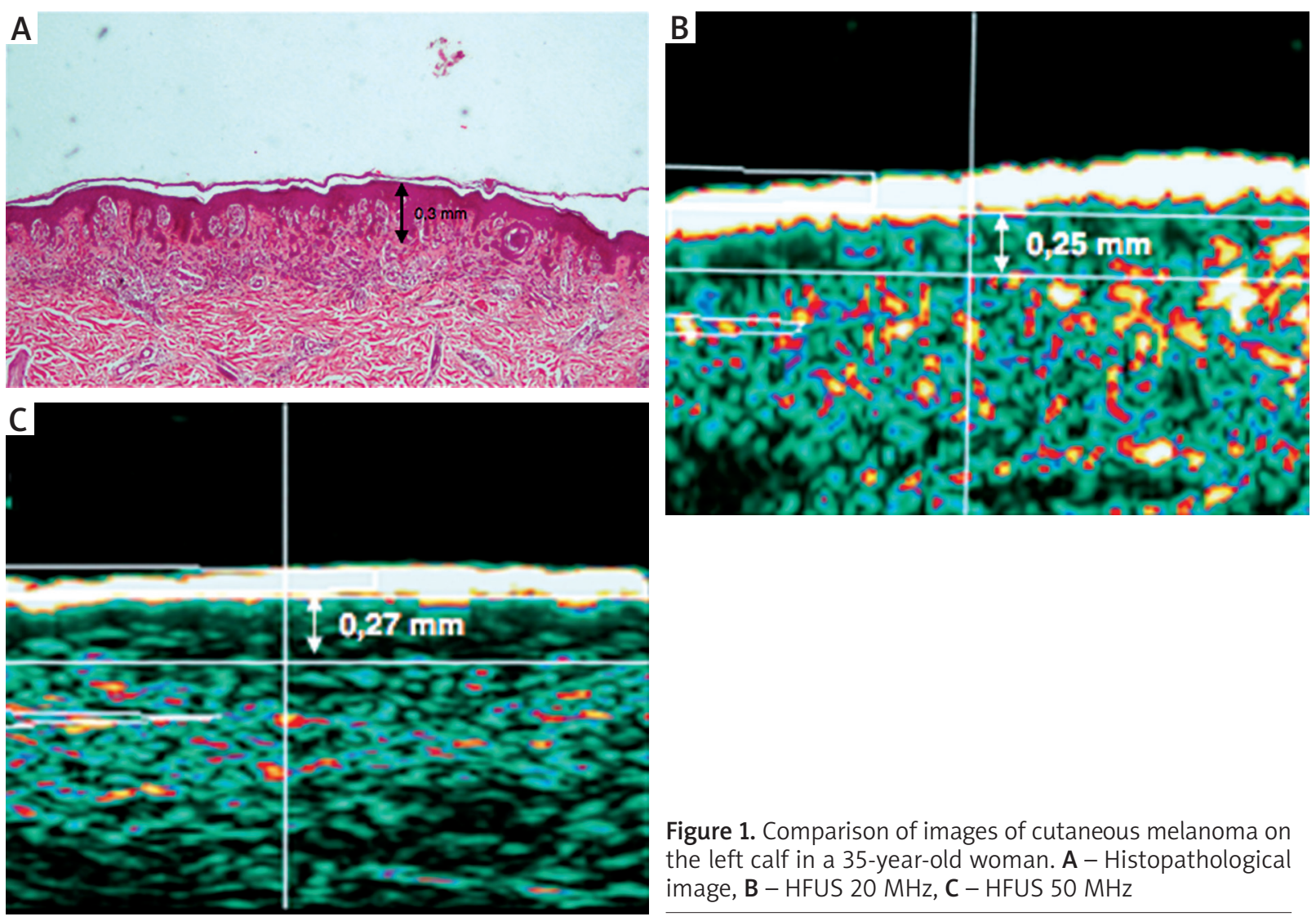

Figure 1. Comparison of images of cutaneous melanoma on the left calf in a 35-year-old woman. A - Histopathological image, B - HFUS $20 \mathrm{MHz}$, C - HFUS $50 \mathrm{MHz}$

as less than or equal to $1 \mathrm{~mm}$ was the same in the case of both probes $(n=15)$. The correlation between the thickness on Breslow's scale and the thickness measured by ultrasound scanning at $20 \mathrm{MHz}$ and $50 \mathrm{MHz}$ was statistically significant and was 0.759 and 0.734 , respectively $(p<0.01)$ (Table 5). The correlation between the thickness on Clark's scale and the thickness measured with the $20 \mathrm{MHz}$ and $50 \mathrm{MHz}$ ultrasound was statistically significant $(p<0.05)$. The linear regression between Breslow's scale and the $50 \mathrm{MHz}$ probe was 0.575, and that between Breslow's scale and the $20 \mathrm{MHz}$ probe was 0.512 (Figure 3). There were no statistically significant differences in measurements with 20 and $50 \mathrm{MHz}$ probes.

\section{Ultrasonography - histopathology correlation}

In the case of 11 lesions of the melanoma type, the thickness obtained in HFUS was higher than that obtained in histopathological examination (HP), and in the case of 4 lesions, the thickness in HFUS was lower than that obtained in HP. In the case of 4 melanomas, identical results were obtained in the histopathological examination and in the HFUS for a margin of $\leq 0.05 \mathrm{~mm}$, of which in the case of one lesion, the result was identical in the study using a $20 \mathrm{MHz}$ probe and the result was overestimated in relation to histopathology using the $50 \mathrm{MHz}$ probe.
Table 1. Surgical margin recommendations for primary cutaneous melanoma according to American Academy of Dermatology [19]

\begin{tabular}{lc}
\hline Tumor thickness & Clinically measured surgical margin $[\mathrm{cm}]$ \\
\hline In situ & $0.5-1.0$ \\
\hline$\leq 1.00$ & 1 \\
\hline $1.01-2.00$ & $1-2$ \\
\hline$\geq 2.01$ & 2 \\
\hline
\end{tabular}

Three of the 4 underestimated lesions were T1NOMO melanomas and were also classified in the same manner in the 20 and $50 \mathrm{MHz}$ HFUS. One underestimated lesion of $2.00 \mathrm{~mm}$ thickness in the histopathological examination was also assigned to the correct group. Despite the underestimation of this melanoma thickness in USG, the obtained value was close enough to the actual thickness to allow it to be included in the correct group according to Breslow's scale. Thicknesses of 1.94 and $1.89 \mathrm{~mm}$ were obtained in the HFUS examination for the 20 and $50 \mathrm{MHz}$ probes, respectively. One of the lesions removed from the skin of the back was 2.4 and $2.1 \mathrm{~mm}$ thick, respectively, for 20 and $50 \mathrm{MHz}$ US, and in histopathological examination the thickness of that melanoma was 

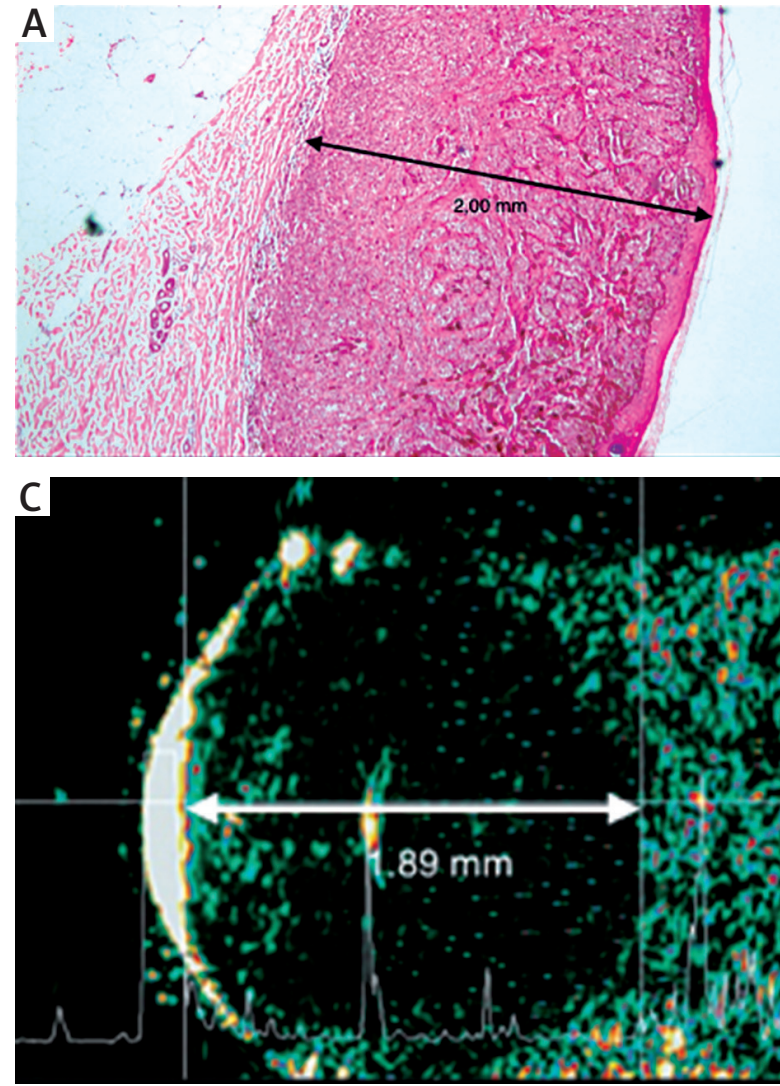

determined to be $0.8 \mathrm{~mm}$ with a present inflammatory infiltrate.

In the case of 4 lesions with a thickness overestimated in the histopathological description, a large inflammatory infiltration was noted, for 2 lesions the infiltration was moderate, for 4 small, and in 1 melanoma it was within the complex nevus. Six of the 10 lesions were located within the torso. The above factors may have contributed to the overestimation of the thickness in the sonographic examination.

\section{Discussion}

The $20 \mathrm{MHz}$ high frequency probe is a good tool for pre-operative evaluation of melanoma lesions. The research carried out so far, as well as the results presented above, seem to confirm this thesis [3]. This is particularly interesting because up to now the $20 \mathrm{MHz}$ probe has been recommended in the evaluation of nodular lesions due to the resolution and depth of measurement it offers (resolution 60 to 150-200 microns, depth of $14 \mathrm{~mm}$ to $23 \mathrm{~mm}$ ). In turn, the $50 \mathrm{MHz}$ probe was preferred for thin, superficial, subcuticular lesions (resolution 30 to 60 microns, $3 \mathrm{~mm}$ penetration). The limitation of the sonographic examination is the inability to accurately and unambiguously distinguish inflammatory infiltrate located in the lower pole of the lesion, which is visible

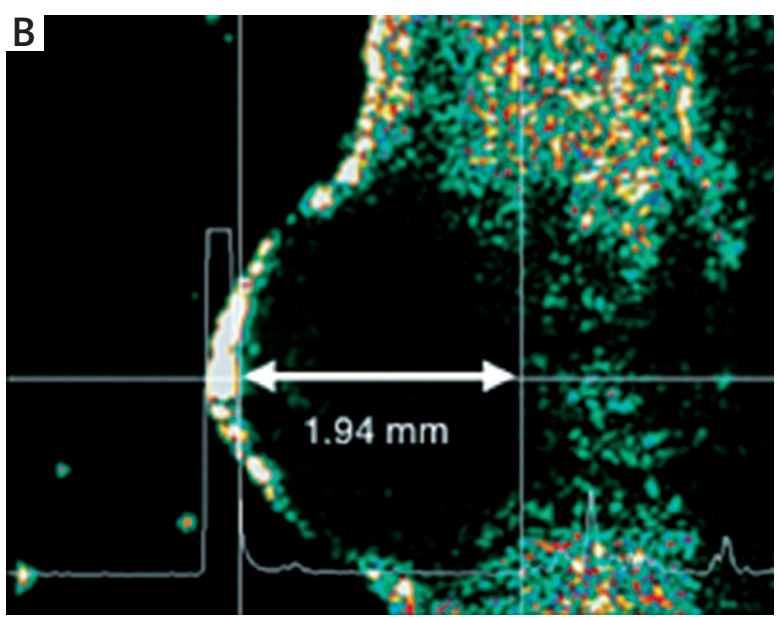

Figure 2. Comparison of images of cutaneous melanoma on the left arm in a 61-year-old woman. A - Histopathological image, B - HFUS $20 \mathrm{MHz}, \mathrm{C}$ - HFUS $50 \mathrm{MHz}$

as a hypoechoic structure which may affect the obtained results [14].

The results of examinations $(n=22)$ performed in the years 1989-2009 using probes with the frequency 7.5-100 MHz were analyzed in the study of Jasaitiene et al. [3]. The largest number of examinations was carried out using a $20 \mathrm{MHz}$ probe $(n=17)$. In each of the analyzed studies, a positive correlation was found $(r=$ 0.76 to 0.99 ) between the thickness of the melanoma obtained in the HFUS examination expressed in $\mathrm{mm}$ and the thickness obtained in the HP test also expressed in $\mathrm{mm}$, whose value depended on the frequency of the applied probe. Analyzing the collected data, the authors determined the correlation between MM measurements obtained in the histopathological and sonographic examination as good or excellent for probes above $20 \mathrm{MHz}$ for MM with a thickness below $1.0 \mathrm{~mm}$, which agrees with the data obtained in our study. It cannot be excluded that the observed overestimation of the thickness of the lesion in the HFUS examination compared to the histopathological examination could be due to the presence of inflammatory infiltration, which in the sonographic examination is hardly visible and distinguishing it from tumor tissue is difficult and sometimes even impossible.

In our study, in the case of 4 lesions with overestimated thickness in the histopathological description, a large inflammatory infiltration was noted, for 2 infil- 
Table 2. Occurrence of melanoma due to sex and age

\begin{tabular}{lcccc}
\hline Sex & $\boldsymbol{N}$ & $\%$ & Mean age & Standard deviation \\
\hline Male & 13 & 68.4 & 55.31 & 16.73 \\
\hline Female & 6 & 31.6 & 58.50 & 10.17 \\
\hline In total & 19 & 100.0 & & \\
\hline Descriptive statistics & Age [years] \\
\hline$N$ & 19 & \\
\hline Mean & 58.31 & \\
\hline Median & 58.00 & \\
\hline Standard deviation & 14.75 \\
\hline Minimum & 26.00 \\
\hline Maximum & 85.00 \\
\hline
\end{tabular}

Table 3. Breslow's scale

\begin{tabular}{lcc}
\hline Descriptive statistics & Breslow's scale [mm] \\
\hline$N$ & \multicolumn{2}{c}{19} \\
\hline Mean & \multicolumn{2}{c}{0.56} \\
\hline Median & \multicolumn{2}{c}{0.40} \\
\hline Standard deviation & \multicolumn{2}{c}{0.47} \\
\hline Minimum & \multicolumn{2}{c}{0.1} \\
\hline Maximum & \multicolumn{2}{c}{2.00} \\
\hline Breslow's scale & $N$ & $\%$ \\
\hline Grade I (<0.75 mm) & 14 & 73.7 \\
\hline Grade II $(0.76-1.50 \mathrm{~mm})$ & 4 & 21.1 \\
\hline Grade III (1.51-2.25 mm) & 1 & \multicolumn{2}{c}{5.3} \\
\hline Grade IV $(2.26-3.00 \mathrm{~mm})$ & 0 & 0 \\
\hline Grade V $(>3.01 \mathrm{~mm})$ & 0 & 0 \\
\hline In total & 19 & 100.0 \\
\hline
\end{tabular}

Table 4. Clark's level

\begin{tabular}{lcc}
\hline Clark's staging & $\boldsymbol{N}$ & $\%$ \\
\hline Level I & 9 & 47.4 \\
\hline Level II & 7 & 36.8 \\
\hline Level III & 1 & 5.3 \\
\hline Level IV & 1 & 5.3 \\
\hline Level V & 1 & 5.3 \\
\hline In total & 19 & 100.0 \\
\hline Mitosis & $\boldsymbol{N}$ & $\%$ \\
\hline $0 / 10$ & 15 & 78.9 \\
\hline $1 / 10$ & 2 & 10.5 \\
\hline $2 / 10$ & 1 & 5.3 \\
\hline $5 / 10$ & 1 & 5.3 \\
\hline In total & 19 & 100.0 \\
\hline
\end{tabular}

Table 5. Analysis of ultrasonographic data

\begin{tabular}{|c|c|c|c|}
\hline Descriptive statistics & \multicolumn{2}{|c|}{$\begin{array}{l}\text { Thickness in USG } \\
20 \mathrm{MHz}[\mathrm{mm}]\end{array}$} & $\begin{array}{l}\text { Thickness in USG } \\
50 \mathrm{MHz}[\mathrm{mm}]\end{array}$ \\
\hline$N$ & \multicolumn{2}{|r|}{19.00} & 19.00 \\
\hline Mean & \multicolumn{2}{|r|}{0.74} & 0.74 \\
\hline Median & \multicolumn{2}{|r|}{0.47} & 0.58 \\
\hline Standard deviation & \multicolumn{2}{|r|}{0.61} & 0.56 \\
\hline Minimum & \multicolumn{2}{|r|}{0.00} & 0.00 \\
\hline Maximum & \multicolumn{2}{|r|}{2.40} & 2.10 \\
\hline \multirow[t]{2}{*}{ Thickness } & \multicolumn{2}{|c|}{ USG $20 \mathrm{MHz}$} & USG $50 \mathrm{MHz}$ \\
\hline & $N$ & $\%$ & $\%$ \\
\hline$\leq 1 \mathrm{~mm}$ & 15 & 78.9 & 78.9 \\
\hline$>1 \mathrm{~mm}$ & 4 & 21.1 & 21.1 \\
\hline In total & 19 & 100.0 & 100.0 \\
\hline \multirow[t]{2}{*}{ USG } & \multicolumn{3}{|c|}{ Breslow's scale } \\
\hline & \multicolumn{2}{|c|}{ Rho } & $P$-value \\
\hline $20 \mathrm{MHz}$ & \multicolumn{2}{|c|}{0.759} & $<0.001$ \\
\hline $50 \mathrm{MHz}$ & \multicolumn{2}{|c|}{0.734} & $<0.001$ \\
\hline
\end{tabular}

tration was moderate, for 4 small and 1 melanoma was within the compound nevus. Additionally, the differences observed in the study may be affected by the shrinkage of the preparation after placing it in the formalin solution used for transport, as well as subjecting it to a treatment aimed at its final placement in the paraffin block. Blasco-Morente et al. demonstrated that the shrinkage of the preparation is $9.5 \%$ for the thickness $(p<0.01)$ and $17.0 \%$ for the width $(p<0.01)$ and is the strongest for lesions cut out within the torso [15]. In our study, 6 out of 10 overestimated lesions were located within the torso.

Evaluation of melanoma thickness is particularly important in both treatment and assessment of patient prognosis. In the studies published so far, a correlation between the thickness of melanoma measured by sonography and that obtained in histopathological examination was found [10, 16-18]. The results of previous studies carried out by the cited authors agree with the results of our study and prove that pre-operative evaluation of melanoma thickness is possible using either a 20 or $50 \mathrm{MHz}$ probe. Moreover, the HFUS examination is standard in pre-operative evaluation of melanoma in Germany and France, especially in determination of resection margins $[12,18,19]$.

Despite numerous important data contributing to the diagnostic test, high resolution ultrasound examination is characterized by numerous limitations. First of all, the lack of sufficiently good resolution and low image quality do not allow for its accurate interpretation. This prevents the use of HFUS as a differential diagnostic tool. Secondly, it is a subjective examination. It means that the researcher requires specialist training in this field 
A
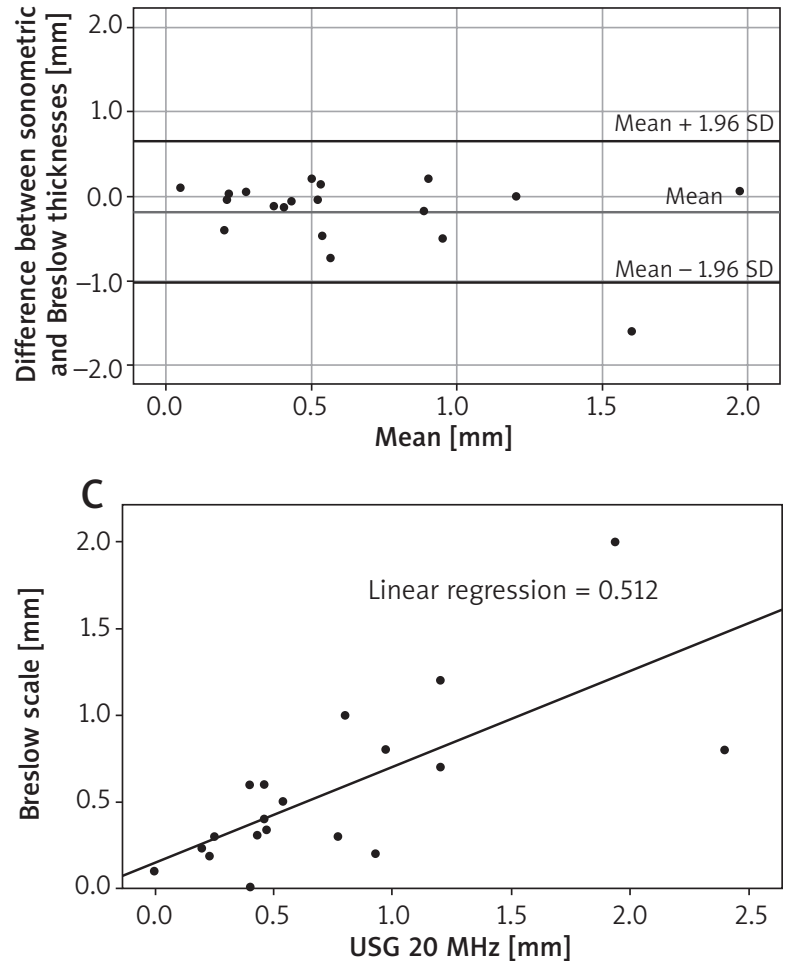

B
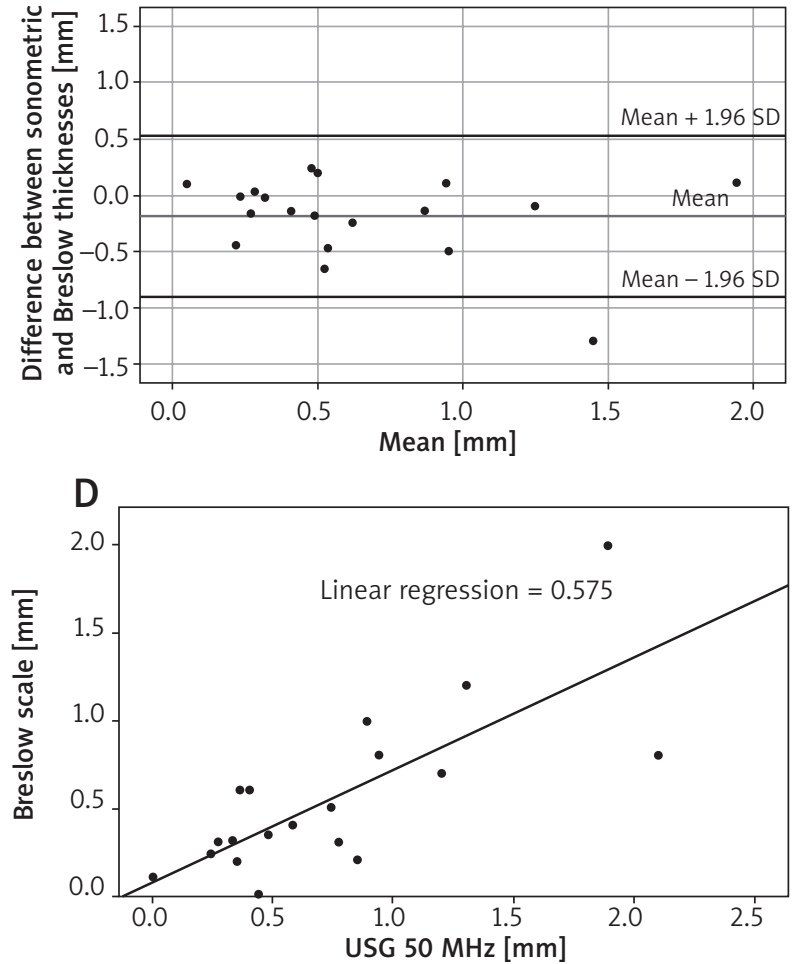

Figure 3. A - Bland-Altman plot showing the difference between the thickness of melanomas measured in $\mathrm{mm}$ on Breslow's scale and the $20 \mathrm{MHz}$ probe. B - Bland-Altman plot showing the difference between the thickness of melanomas measured in $\mathrm{mm}$ on Breslow's scale and the $50 \mathrm{MHz}$ probe. C - Linear relation between the $20 \mathrm{MHz}$ probe and the thickness expressed in $\mathrm{mm}$ on Breslow's scale. $\mathrm{D}$ - Linear relation between the $50 \mathrm{MHz}$ probe and the thickness expressed in $\mathrm{mm}$ on Breslow's scale

and starting the examination using HFUS should have minimal experience acquired under the supervision of a specialist.

Despite the development of numerous new imaging methods, histopathological examination continues to be the gold standard in MM assessment and allows one to determine parameters such as the thickness according to Breslow's scale, infiltration on Clark's scale, number of mitoses in the field of view, presence of ulcer, inflammatory infiltration at the base, infiltration of blood and lymph vessels. In addition, histopathology allows the creation of specific staining, which further increases the accuracy of the diagnosis [1].

\section{Conclusions}

No differences in determination of melanoma thickness using 50 and $20 \mathrm{MHz}$ probes were observed in our study. Similar thickness values of cancer lesions were obtained in both cases.

A statistically significant correlation was found between the thickness of melanoma obtained in the sonographic examination and Breslow's scale using both examined probes. This indicates that both probes can be used in the evaluation of MM. Melanoma thickness above $1 \mathrm{~mm}$ has no effect on the effectiveness of its thickness assessment with HFUS.

The HFUS examination is applicable only in the evaluation of MM thickness. No other feature and parameter can be determined using this method.

It seems necessary to develop standardization of the method using HFUS in pre-operative evaluation of melanoma.

\section{Conflict of interest}

The authors declare no conflict of interest.

\section{References}

1. Nagore E, Oliver V, Botella-Estrada R, et al. Prognostic factors in localized invasive cutaneous melanoma: high value of mitotic rate, vascular invasion and microscopic satellitosis. Melanoma Res 2005; 15: 169-77.

2. Dickson PV, Gershenwald JE. Staging and prognosis of cutaneous melanoma. Surg Oncol Clin N Am 2011; 20: 1-17.

3. Jasaitiene D, Valiukeviciene S, Linkeviciute G, et al. Principles of high-frequency ultrasonography for investigation of skin pathology. J Eur Adv Venereol 2011; 25: 375-82. 
4. Music MM, Hertl K, Kadivec M, et al. Pre-operative ultrasound with a $12-15 \mathrm{MHz}$ linear probe reliably differentiates between melanoma thicker and thinner than $1 \mathrm{~mm}$. J Eur Adv Venereol 2010; 24: 1105-8.

5. Kaikaris V, Samsanavicius D, Maslauskas K, et al. Measurement of melanoma thickness - comparison of two methods: ultrasound versus morphology. J Plast Reconstr Aesthet Surg 2011; 64: 796-802.

6. Hinz T, Ehler LK, Voth H, et al. Assessment of tumor thickness in melanocytic skin lesions: comparison of optical coherence tomography, 20-MHz ultrasound and histopathology. Dermatology 2011; 223: 161-8.

7. Crisan M, Crisan D, Sannino G, et al. Ultrasonographic staging of cutaneous malignant tumors: an ultrasonographic depth index. Arch Dermatol Res 2013; 305: 305-13.

8. Fernández Canedo I, de Troya Martín M, Fúnez Liébana R, et al. Preoperative 15-MHz ultrasound assessment of tumor thickness in malignant melanoma. Actas Dermosifiliogr 2013; 104: 227-31.

9. Meyer N, Lauwers-Cances V, Lourari S, et al. High-frequency ultrasonography but not 930-nm optical coherence tomography reliably evaluates melanoma thickness in vivo: a prospective validation study. Br I Dermatol 2014; 171: 799-805.

10. Wang SQ, Hashemi P. Noninvasive imaging technologies in the diagnosis of melanoma. Semin Cutan Med Surg 2010; 29: 174-84.

11. Lassau N, Lamuraglia M, Koscielny S, et al. Prognostic value of angiogenesis evaluated with high-frequency and colour Doppler sonography for preoperative assessment of primary cutaneous melanomas: correlation with recurrence after a 5 year follow-up period. Cancer Imaging 2006; 6: 24-9.

12. Machet L, Samimi M, Georgesco G, et al. High Resolution Ultrasound Imaging of Melanocytic and Other Pigmented Lesions of the Skin. Ultrasound Imaging, Mr Masayuki Tanabe (Ed.), ISBN: 978-953-307-239-5, InTech, Available from: https://www.intechopen.com/books/ultrasound-imaging/ high-resolution-ultrasound-imaging-of-melanocytic-andother-pigmented-lesions-of-the-skin

13. Surgical margin recommendations for primary cutaneous melanoma according to American Academy of Dermatology. Retrieved from: https://www.aad.org/practicecenter/quality/ clinical-guidelines/melanoma/surgical-management

14. Dill-Müller D, Maschke J. Ultrasonography in dermatology. J Dtsch Dermatol Ges 2007; 5: 689-707.

15. Blasco-Morente G, Garrido-Colmenero C, Pérez-López I, et al. Study of shrinkage of cutaneous surgical specimens. J Cutan Pathol 2015; 42: 253-7.

16. Guitera P, Li LX, Crotty K, et al. Melanoma histological Breslow thickness predicted by $75-\mathrm{MHz}$ ultrasonography. Br J Dermatol 2008; 159: 364-9.

17. Machet L, Belot V, Naouri M, et al. Preoperative measurement of thickness of cutaneous melanoma using high-resolution $20 \mathrm{MHz}$ ultrasound imaging: a monocenter prospective study and systematic review of the literature. Ultrasound Med Biol 2009; 35: 1411-20.

18. Kozárová A, Kozár M, Tonhajzerová I, et al. The value of highfrequency $20 \mathrm{MHz}$ ultrasonography for preoperative measurement of cutaneous melanoma thickness. Acta Dermatovenerol Croat 2018; 26: 15-20.

19. Polańska A, Dańczak-Pazdrowska A, Jałowska M, et al. Current applications of high-frequency ultrasonography in dermatology. Adv Dermatol Allergol 2017; 34: 535-42. 